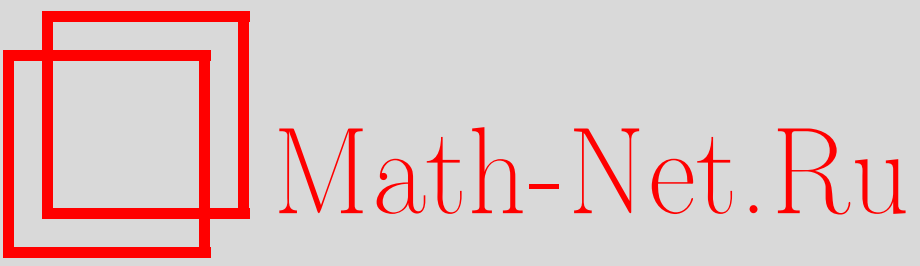

P. Джакив, Гидродинамические профили и интегралы движения из $d$-бран, TMФ, 2000, том 124, номер 2, 215-226

DOI: https://doi.org/10.4213/tmf634

Использование Общероссийского математического портала Math-Net.Ru подразумевает, что вы прочитали и согласны с пользовательским соглашением

http://www.mathnet.ru/rus/agreement

Параметры загрузки:

IP : 54.197 .217 .227

26 апреля 2023 г., $07: 57: 24$ 
ТЕОРЕТИЧЕСКАЯ

И МАТЕМАТИЧЕСКАЯ

ФИЗИКА

Том 124, № 2

август, 2000

(C) $2000 \mathrm{r}$.

Р. Джакив*

\section{ГИДРОДИНАМИЧЕСКИЕ ПРОФИЛИ И ИНТЕГРАЛЫ ДВИЖЕНИЯ ИЗ $d$-БРАН ${ }^{1)}$}

Различные гидродинамические системы, подчиняющиеся нелинейным дифференциальным уравнениям, имеют скрытую динамическую симметрию Пуанкаре в высших размерностях, поскольку они следуют из действия Намбу-Гото. По той же причине существуют и преобразования эквивалентности между различными моделями. Эти взаимосвязи обсуждаются и формулируются в виде простой диаграммы.

\section{ВВЕДЕНИЕ}

Настоящая работа основана на лекции, прочитанной в Институте теоретической физики им. Н.Н. Боголюбова в честь 90-летней годовшины выдаюшегося математика и физика Н.Н. Боголюбова, долгое время работавшего в Киеве. Исследования в области теории струн в настоящее время вдохновляют оживленный обмен мнениями между математиками и физиками, которому Боголюбов был бы рад оказаться свидетелем. Его деятельность охватывала теорию поля, нелинейные системы и кинетическую теорию. В данной работе, посвященной его памяти, описываются взаимосвязи именно между этими областями.

Мы обсуждаем несколько нелинейных уравнений математической физики в пространстве произвольной размерности, обладающих замечательными скрытыми симметриями и неожиданными интегралами движения, позволяюшими строить решения и осуществлять полное интегрирование. Более того, между уравнениями имеются связи, позволяющие отображать одно уравнение на другое. Используя современный язык, можно сказать, что имеются "дуальности", связывающие совершенно разные модели. Кроме того, мы описываем, каким образом все эти свойства следуют из "Ur-формулировки" в терминах протяженных объектов ( $d$-бран) в высших размерностях. Нелинейные уравнения, которые мы обсуждаем, сушествовали раньше и хорошо известны; они непостроены специально для иллюстрации теории. Наблюдения относительно этих уравнений

\footnotetext{
1) Расширенная версия пленарного доклада Р. Джакива на мемориальной Боголюбовской конференции 1999 г.
}

${ }^{*}$ Center for Theoretical Physics, Massachusetts Institute of Technology, Cambridge, Mass., USA 
в основном являются новыми, хотя некоторые результаты в пространствах низкой размерности были известны ранее. Описанная здесь работа была выполнена в сотрудничестве с Базейа $[1,2]$ и Полихронакосом [3]; она основывается на результатах, исходно полученных в этой области Бордеманном и Хоппе [4], а также Джевицки [5].

\section{1. НЕРЕЛЯТИВИСТСКАЯ МОДЕЛЬ}

Первое уравнение, которое мы рассмотрим, описьвает движение нерелятивистской жидкости в пространстве размерности $d$, т.е. в $(d, 1)$-мерном пространстве-времени. Введем плотность вешества жидкости $\rho(t, \mathbf{r})$, локальную скорость $\mathbf{v}(t, \mathbf{r})$ и ток $\mathbf{j}(t, \mathbf{r})=$ $\mathbf{v}(t, \mathbf{r}) \rho(t, \mathbf{r})$, который связан с плотностью уравнением непрерывности

$$
\dot{\rho}+\nabla \cdot(\mathbf{v} \rho)=0
$$

где точка над символом обозначает дифференцирование по времени. Скорость удовлетворяет уравнению Эйлера, связывающему ее производную по времени с силой (на единицу объема), а именно с градиентом давления $P$, деленным на $\rho$ :

$$
\dot{\mathbf{v}}+\mathbf{v} \cdot \nabla \mathbf{v}=\mathbf{f}=-\frac{\nabla P}{\rho} .
$$

В нерелятивистском случае нас интересует сила $\mathbf{f}$ весьма специального вида со следуюшими свойствами:

1) сила $f$ возникает из давления $P$, которое является функцией только $\rho$ (что соответствует изэнтропийному течению);

2 ) давление $P$ является политропной формой ( $P$ пропорционально степени $\rho)$;

3) конкретный степенной закон является законом обратной пропорциональности, т.е.

$$
P(\rho)=-\frac{2 \lambda}{m \rho}
$$

Такое явление называется газом Чаплыгина и соответствует скорости звука $\sqrt{2 \lambda / m} / \rho$ (поэтому мы выбираем $\lambda \geqslant 0)$ и энтальпии $\lambda / m \rho^{2}(m-$ масса).

Уравнение Эйлера для газа Чаплыгина имеет вид

$$
\dot{\mathbf{v}}+\mathbf{v} \cdot \nabla \mathbf{v}=-\frac{2 \lambda}{m} \frac{\nabla \rho}{\rho^{3}}
$$

Поскольку непротиворечивым оказывается поиск безвихревых решений $(\boldsymbol{\nabla} \times \mathbf{v}=0)$, мы запишем $\mathbf{v}=\nabla \theta / m$ и заменим уравнение (3) уравнением Бернулли для потенциала скорости $\theta$ с членом, учитывающим источник, задаваемый энтальпией:

$$
\dot{\theta}+\frac{(\nabla \theta)^{2}}{2 m}=\frac{\lambda}{\rho^{2}} .
$$

Взяв градиент (4), мы получим уравнение (3). В итоге мы изучаем неротационное, изэнтропийное движение газа Чаплыгина. 
Уравнения (1) и (4) допускают формулировку в терминах действия с лагранжианом первого порядка (по времени)

$$
L_{\mathrm{nr}}=\int d r\left[\theta \dot{\rho}-\left(\rho \frac{(\nabla \theta)^{2}}{2 m}+\frac{\lambda}{\rho}\right)\right] .
$$

Действительно, гамильтониан имеет вид

$$
H_{\mathrm{nr}}=\int d r \mathcal{H}_{\mathrm{nr}}=\int d r\left(\rho \frac{(\boldsymbol{\nabla} \theta)^{2}}{2 m}+\frac{\lambda}{\rho}\right),
$$

а каноническая 1-форма

$$
\int d r \theta d \rho
$$

приводит к скобкам Пуассона

$$
\left\{\theta(t, \mathbf{r}), \rho\left(t, \mathbf{r}^{\prime}\right)\right\}=\delta\left(\mathbf{r}-\mathbf{r}^{\prime}\right) .
$$

Легко проверить, что уравнения (1) и (4) представляют собой гамильтонову систему с такой скобкой.

Будучи нерелятивистской, описанная модель имеет соответствуюшую нерелятивистскую симметрию, а именно инвариантность Галилея. Интегралы движения, которые являются следствием теоремы Нетер, порождают инфинитезимальные преобразования Галилея посредством скобок. Перечислим эти преобразования, чтобы ссылаться на них в дальнейшем: временной сдвиг, пространственный сдвиг и врашение действуют на координаты $(t, \mathbf{r})$ очевидным образом, а преобразованные поля $(\rho, \theta)$ вычисляются в преобразованных координатах. Соответствуюшие интегралы движения - это энергия

$$
E=H=\int d r \mathcal{H},
$$

импульс

$$
\mathbf{P}=\int d r \mathcal{P}
$$

и момент импульса

$$
L^{i j}=\int d r\left(r^{i} \mathcal{P}^{j}-r^{j} \mathcal{P}^{i}\right) .
$$

Имеем плотность энергии

$$
\mathcal{H}=\frac{\rho(\nabla \theta)^{2}}{2 m}+\frac{\lambda}{\rho}
$$

и плотность импульса $\mathcal{P}=\rho \boldsymbol{\nabla} \theta$.

Имеются также галилеевские бусты, которые осуществляют преобразование пространственной координаты, соответствующее скорости $\mathbf{u}$

$$
\mathbf{r} \rightarrow \mathbf{R} \equiv \mathbf{r}-t \mathbf{u} .
$$


Поле плотностей преобразуется просто:

$$
\rho(t, \mathbf{r}) \rightarrow \rho_{\mathbf{u}}(t, \mathbf{r})=\rho(t, \mathbf{R}) .
$$

Потенциал скорости претерпевает аффинное преобразование

$$
\theta(t, \mathbf{r}) \rightarrow \theta_{\mathbf{u}}(t, \mathbf{r}) \equiv \theta(t, \mathbf{R})+m\left(\mathbf{u} \cdot \mathbf{r}-\frac{u^{2} t}{2}\right)
$$

вследствие которого скорость подвергается (как и ожидалось) бусту:

$$
\mathbf{v}(t, \mathbf{r}) \rightarrow \mathbf{v}_{\mathbf{u}}(t, \mathbf{r})=\mathbf{v}(t, \mathbf{R})+\mathbf{u}
$$

Соответствующий интеграл движения является генератором буста:

$$
\mathbf{B}=t \mathbf{P}-m \int d r \mathbf{r} \rho .
$$

Кроме того, имеется закон сохранения вешества как следствие инвариантности при сдвигах $\theta$ на константу:

$$
\theta \rightarrow \theta+\text { const }, \quad \rho \rightarrow \rho .
$$

Соответствующий интеграл движения принимает вид

$$
N=\int d r \rho .
$$

Можно проверить, что действие

$$
I_{\mathrm{nr}}=\int d t L_{\mathrm{nr}}
$$

инвариантно относительно всех этих преобразований и поэтому преобразование решения уравнений движения (1) и (4) является снова решением. Все генераторы, которые могут быть получены из лагранжиана (5) с использованием теоремы Нетер, не зависят от времени, что можно проверить, дифференцируя их по времени и вычисляя $(\dot{\rho}, \dot{\theta})$ из уравнений движения (1) и (4).

Заметим, что имеется $(d+1)(d+2) / 2+1$ генераторов, это правильное число для (центрально расширенной) группы Галилея, где задающие расширение 1-коцикл и 2-коцикл отвечают за неоднородный член в уравнении (9) и приводят к генератору $N(11)$.

Газ Чаплыгина замечателен тем, что обладает (в любом числе измерений) и другими симметриями. Во-первых, мы можем изменить масштаб времени:

$$
t \rightarrow T=e^{\omega} t
$$

Поля подвергаются дополнительному и противоположному рескейлингу:

$$
\begin{aligned}
& \theta(t, \mathbf{r}) \rightarrow \theta_{\omega}(t, \mathbf{r})=e^{\omega} \theta(T, \mathbf{r}) \\
& \rho(t, \mathbf{r}) \rightarrow \rho_{\omega}(t, \mathbf{r})=e^{-\omega} \rho(T, \mathbf{r})
\end{aligned}
$$


Не зависимый от времени генератор имеет вид

$$
D=t H-\int d r \rho \theta
$$

Более того, специальным образом зависяший от полей диффеоморфизм, перемешивающий независимые переменные $(t, \mathbf{r})$ и зависимые поля $(\rho, \theta)$, также оставляет действие инвариантным. Преобразование параметризуется $d$-компонентным вектором $\boldsymbol{\omega}$, который действует на координаты следуюшим образом:

$$
\begin{aligned}
& t \rightarrow T=t+\boldsymbol{\omega} \cdot \mathbf{r}+\frac{\omega^{2} \theta(T, \mathbf{R})}{2 m}, \\
& \mathbf{r} \rightarrow \mathbf{R}=\mathbf{r}+\frac{\boldsymbol{\omega} \theta(T, \mathbf{R})}{m}
\end{aligned}
$$

Поля преобразуются согласно закону

$$
\begin{aligned}
& \theta(t, \mathbf{r}) \rightarrow \theta_{\boldsymbol{\omega}}(t, \mathbf{r})=\theta(T, \mathbf{R}), \\
& \rho(t, \mathbf{r}) \rightarrow \rho_{\boldsymbol{\omega}}(t, \mathbf{r})=\frac{\rho(T, \mathbf{R})}{|J|},
\end{aligned}
$$

где $|J|$ - якобиан преобразования:

$$
J=\operatorname{det}\left[\begin{array}{cc}
\frac{\partial T}{\partial t} & \frac{\partial \mathbf{R}}{\partial t} \\
\frac{\partial T}{\partial \mathbf{r}} & \frac{\partial \mathbf{R}}{\partial \mathbf{r}}
\end{array}\right]
$$

$d$-Компонентный векторный генератор имеет вид

$$
\mathbf{G}=\int d r\left\{\mathbf{r} \mathcal{H}-\frac{\theta \mathcal{P}}{m}\right\}
$$

Так же как и в случае традиционных преобразований/симметрий, эти преобразования оставляют действие инвариантным и поэтому переводят решения в новые решения. Дополнительные $d+1$ генераторов $(D, \mathbf{G})$ получаются с использованием теоремы Нетер и не зависят от времени в силу уравнений движения.

Используя канонический коммутатор (7) и явные формулы для генераторов, мы можем вычислить алгебру Ли последних. Как и ожидалось, $(d+1)(d+2) / 2+1$ генераторов Галилея $(8),(10)$ и (11) замыкаются на (расширенную) алгебру Галилея в $(d, 1)$-мерном пространстве-времени. Дополняя их $d+1$ дополнительными генераторами (15) и (20), в итоге получим $(d+2)(d+3) / 2$ генераторов, алгебра которых замыкается на алгебру Пуанкаре в размерности на единицу выше, а именно в $(d+1,1)$-мерном пространстве-времени. Более того, мы установили, что величины $(t, \theta, \mathbf{r})$ преобразуются как компоненты светового конуса $(d+2)$-компонентного лоренцева вектора, причем $t$ играет роль "+"-компоненты, а $\theta$ - роль "-"-компоненты [2]. 


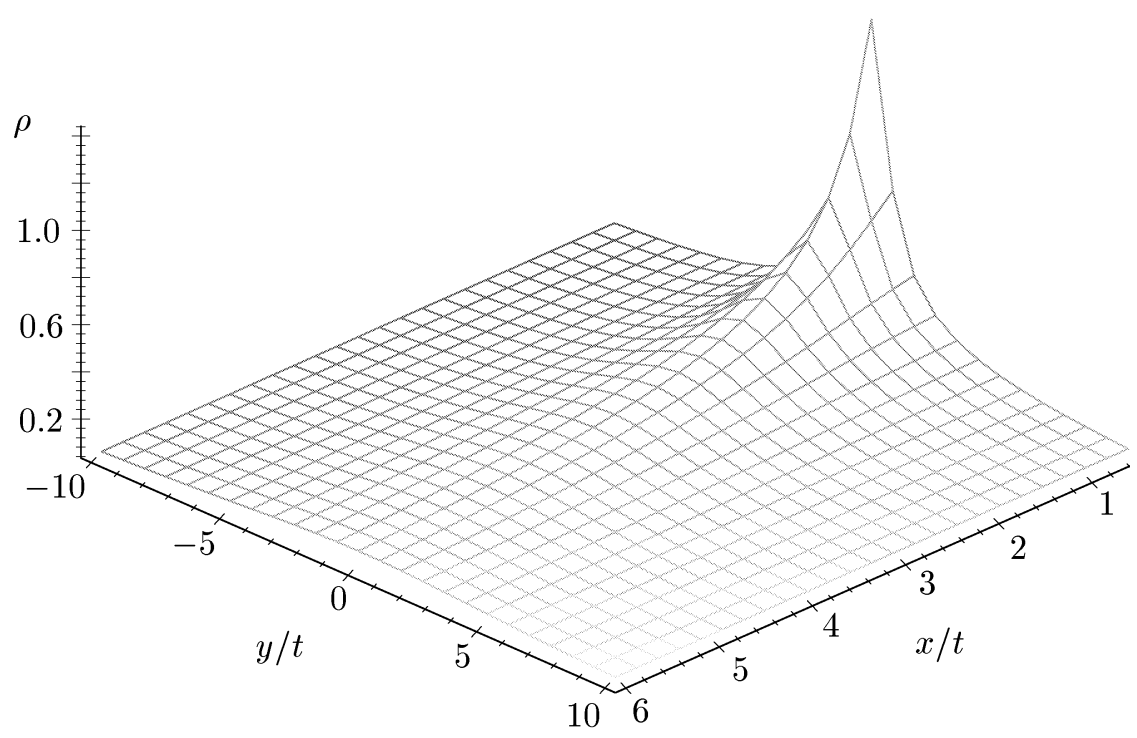

Рис. 1. Исходная плотность $\rho(t, \mathbf{r}) \propto|t| / r$ (для двух пространственных измерений); $\mathbf{r}=(x, y)$.

Таким образом, мы заключаем, что нерелятивистский галилеевски-инвариантный газ Чаплыгина в $(d, 1)$-мерном пространстве-времени обладает скрытой динамической симметрией Пуанкаре, которая присуша $(d+1,1)$-мерному пространству-времени и которая нелинейно реализуется с помошью зависяших от полей диффеоморфизмов.

Мы можем использовать симметрию, для того чтобы порождать новые решения из старых. Конечно, когда преобразования имеют знакомый галилеевский вид, "новые" решения обладают очевидным соответствием со старыми: они подвергаются временной или пространственной трансляции, пространственному врашению или бусту, или $\theta$-сдвигу. Однако, когда преобразования относятся к скрытым симметриям, новые решения принимают новый, неожиданный вид.

Например, простое решение уравнений (1) и (4) выглядит следуюшим образом:

$$
\theta=-\frac{m r^{2}}{2(d-1) t}, \quad \rho=\sqrt{\frac{2 \lambda}{m d}}(d-1) \frac{|t|}{r}, \quad d>1 .
$$

Это соответствует скорости и току

$$
\mathbf{v}=-\frac{1}{(d-1)} \frac{\mathbf{r}}{t}, \quad \mathbf{j}=-\epsilon(t) \sqrt{\frac{2 \lambda}{m d}} \hat{r}, \quad d>1,
$$

где $\epsilon(t)=t /|t|$ - ступенчатая функция. В уравнении $(21)(\theta, \rho)$-профили инвариантны относительно масштабного преобразования времени (12)-(14), однако зависяший от поля диффеоморфизм (16)-(19) радикально меняет решение. Аналитическое выражение 


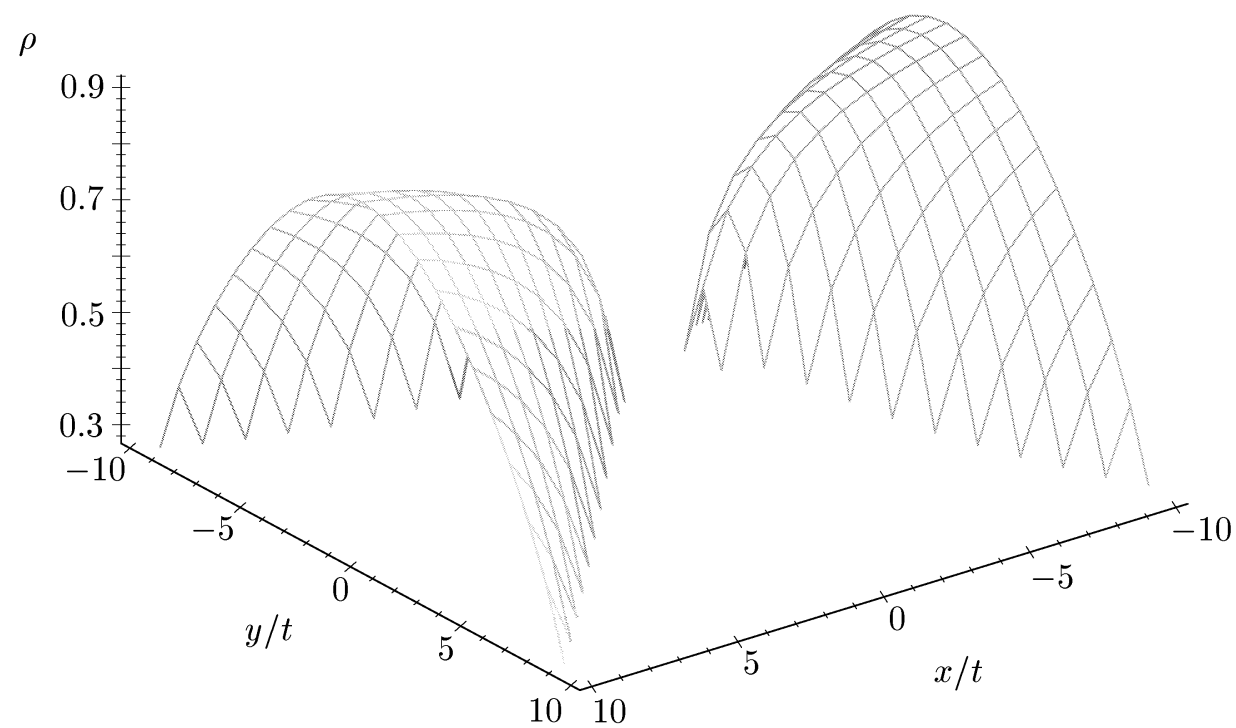

РИс. 2. Преобразованная плотность $\rho(t, \mathbf{r})$.

для преобразованного профиля неинформативно; ситуацию проясняют рисунки. Двумерные решения изображены на рис. 1 и 2: на рис. 1 представлена плотность $\rho$ из уравнения (21), а на рис. 2 - преобразованная плотность $\rho$ (аналитические выражения для преобразованных решений приведены в работе [1]).

В случае одного пространственного измерения уравнения являются полностью интегрируемыми [6]. Намек на это можно усмотреть в специальном решении, где ток демонстрирует солитонный профиль: при $d=1$ имеем

$$
\begin{aligned}
& \theta=-\frac{m}{2 k^{2} t} \operatorname{ch}^{2} k x, \\
& \rho=\sqrt{\frac{2 \lambda}{m}} \frac{k|t|}{\operatorname{ch}^{2} k x}, \\
& j=-\epsilon(t) \sqrt{\frac{2 \lambda}{m}} \operatorname{th} k x .
\end{aligned}
$$

В заключение этого раздела отметим, что наши новые симметрии действуют равным образом и на модель без взаимодействия $(\lambda=0)$, для которой полное решение можно в действительности задать в терминах начальных условий для $\rho$ и $\mathbf{v}$ :

$$
\left.\rho(t, \mathbf{r})\right|_{t=0}=\rho_{0}(\mathbf{r}),\left.\quad \mathbf{v}(t, \mathbf{r})\right|_{t=0}=\mathbf{v}_{0}(\mathbf{r})
$$

Определим “запаздываюшее” положение $\mathbf{q}(t, \mathbf{r})$ как

$$
\mathbf{q}+t \mathbf{v}_{0}(\mathbf{q})=\mathbf{r} .
$$


Решение уравнений (1), (2) без взаимодействия имеет вид

$$
\mathbf{v}(t, \mathbf{r})=\mathbf{v}_{0}(\mathbf{q}), \quad \rho(t, \mathbf{r})=\rho_{0}(\mathbf{q})\left|\operatorname{det} \frac{\partial q^{j}}{\partial r^{j}}\right| .
$$

При $\lambda \neq 0$ можно исключить $\rho$ в пользу $\theta$ и записать лагранжиан, эквивалентный (5), в виде

$$
L_{\lambda}=-2 \lambda \int d r \sqrt{\dot{\theta}+\frac{(\nabla \theta)^{2}}{2 m}} .
$$

Отметим, что уравнения движения для $\theta$ более не содержат $\lambda$, которая служит просто для нормировки лагранжиана. Несмотря на свой необычный вид уравнение (25) определяет галилеевски-инвариантную теорию, обладающую также дополнительными симметриями (13) и (18).

\section{2. РЕЛЯТИВИСТСКАЯ МОДЕЛЬ}

Можно предложить релятивистское обобшение газа Чаплыгина. Соответствуюший лагранжиан в пространстве размерности $d$ имеет вид

$$
\begin{aligned}
L_{\mathrm{r}} & =\int d r\left[\theta \dot{\rho}-\left(\sqrt{\rho^{2} c^{2}+a^{2}} \sqrt{m^{2} c^{2}+(\boldsymbol{\nabla} \theta)^{2}}\right)\right]= \\
& =\int d r\left[\theta \dot{\rho}-\mathcal{H}_{\mathrm{r}}\right]
\end{aligned}
$$

где $a$ является мерой взаимодействия. При $a=0$ получаем релятивистское обобшение модели (5) с $\lambda=0$. Удерживая $a$ и полагая $c \rightarrow \infty$, находим, что

$$
L_{\mathrm{r}}=-N m c^{2}+\left.L_{\mathrm{nr}}\right|_{\lambda=m a^{2} / 2} .
$$

Хотя это и не сразу очевидно, $L_{\mathrm{r}}$ является лоренц- и пуанкаре-инвариантом в $(d, 1)$-мерном пространстве-времени (см. ниже) и вещество также сохраняется. Таким образом, имеется $(d+1)(d+2) / 2+1$ генераторов, где первое слагаемое дает число генераторов Пуанкаре и “+1” соответствует генератору $N$.

При $a=0$ модель является свободной и элементарной; имеется полное решение. Действительно, уравнения движения принимают вид (1) и (2) (с равным нулю f). Однако $\mathbf{v}$ равно не $\boldsymbol{\nabla} \theta / m$, а $c \boldsymbol{\nabla} \theta / \sqrt{m^{2} c^{2}+(\boldsymbol{\nabla} \theta)^{2}}$ и $\boldsymbol{\nabla} \theta / m=\mathbf{v} / \sqrt{1-v^{2} / c^{2}}$. Поэтому решения принимают тот же вид, что в уравнениях $(22)-(24)$.

Как и в нерелятивистском случае, при $a \neq 0$ можно исключить $\rho$, что приводит к лагранжиану, подобному лагранжиану модели Борна-Инфельда

$$
L_{a}=-a \int d r \sqrt{m^{2} c^{2}-\left(\partial_{\mu} \theta\right)^{2}}
$$

Как и раньше, сила взаимодействия исчезает из уравнений движения для $\theta$ и служит просто для нормировки лагранжиана. Явная пуанкаре-симметрия теперь становится очевидной, и ее генераторы можно построить обычным образом из тензора энергии-импульса для (27). 
Вследствие аналогий с нерелятивистской моделью неудивительно, что релятивистская модель также обладает дополнительными скрытыми симметриями, оставляюшими действие инвариантным и переводяшими решения в новые решения. Можно репараметризовать время с помощью зависящего от полей преобразования, содержащего скаляр $\omega$ :

$$
\begin{aligned}
& t \rightarrow T=\frac{t}{\operatorname{ch} m c^{2} \omega}+\frac{\theta(T, \mathbf{r})}{m c^{2}} \operatorname{th} m c^{2} \omega \\
& \theta(t, \mathbf{r}) \rightarrow \theta_{\omega}(t, \mathbf{r})=\frac{\theta(T, \mathbf{r})}{\operatorname{ch} m c^{2} \omega}-m c^{2} \operatorname{th} m c^{2} \omega
\end{aligned}
$$

Соответствуюший сохраняюшийся генератор имеет вид

$$
D=\int d r\left(m^{2} c^{4} t \rho+\theta \mathcal{H}_{\mathrm{r}}\right) .
$$

Кроме того, имеется еше и пространственная репараметризация, определяемая $d$-компонентным вектором $\boldsymbol{\omega}(\omega \equiv|\boldsymbol{\omega}|)$,

$$
\begin{array}{r}
\mathbf{r} \rightarrow \mathbf{R}=\mathbf{r}-\boldsymbol{\omega} \theta(t, \mathbf{R}) \frac{\operatorname{tg} m c \omega}{m c \omega}+\boldsymbol{\omega} \boldsymbol{\omega} \cdot \mathbf{r} \frac{1-\cos m c \omega}{\omega^{2} \cos m c \omega} \\
\theta(t, \mathbf{r}) \rightarrow \theta_{\boldsymbol{\omega}}(t, \mathbf{r})=\frac{\theta(t, \mathbf{R})}{\cos m c \omega}-m c \boldsymbol{\omega} \cdot \mathbf{r} \frac{\operatorname{tg} m c \omega}{\omega}
\end{array}
$$

с сохраняющимся векторным генератором

$$
\mathbf{G}=\int d r\left(m^{2} c^{2} r \rho+\theta \mathcal{P}\right) .
$$

Указан только закон преобразования для $\theta$; закон преобразования для $\rho$ можно вывести из уравнения движения для $\theta$, вытекаюшего из лагранжиана (26).

Дополнительные симметрии дают еше $d+1$ генераторов, которые дополняют описанные ранее $(d+1)(d+2) / 2+1$ генераторов до общего числа $(d+2)(d+3) / 2$, которое как раз и соответствует группе Пуанкаре в $(d+1,1)$-мерном пространстве-времени. В самом деле, вычисляя канонические скобки Ли для всех генераторов, мы находим, что вся совокупность генераторов замыкается на алгебру Пуанкаре в $(d+1,1)$-мерии. Вычисление основано на скобке (7), имеюшей тот же вид, что и в нерелятивистском случае (поскольку каноническая 1-форма (26) совпадает с формой (5) и дается формулой (6)). Более того, множество величин $(t, \theta, \mathbf{r})$ преобразуется как $(d+2)$-компонентньй лоренцев вектор в декартовых координатах [2].

Как и в нерелятивистском случае, можно использовать преобразования дополнительных скрытых симметрий, для того чтобы отображать решения в новые решения с различными свойствами. Кроме того, можно использовать связь между релятивистским и нерелятивистским случаями, чтобы получить решения задачи о газе Чапльгина путем взятия предела решения Борна-Инфельда при $c \rightarrow \infty$ [3]. Модель Борна-Инфельда является полностью интегрируемой в одном пространственном измерении [7]. 


\section{3. ОБШАЯ РОДОСЛОВНАЯ}

"Скрытые" симметрии и соответствуюшие им бессистемные законы преобразований можно систематизировать, рассматривая действие Намбу-Гото для $d$-браны в $(d+1)$ мерном пространстве, движушейся в $(d+1,1)$-мерном пространстве-времени ( $d$-брана является $d$-мерным протяженным объектом: 1-брана - струна, 2-брана - мембрана и т.д., $d$-брана в $(d+1)$-мерном пространстве разбивает пространство на две компоненты).

Действие Намбу-Гото имеет вид

$$
I_{\mathrm{NG}}=\int d \phi^{0} d \phi^{1} \ldots d \phi^{d} \sqrt{G},
$$

где

$$
G=(-1)^{d} \operatorname{det} \frac{\partial X^{\mu}}{\partial \phi^{\alpha}} \frac{\partial X_{\mu}}{\partial \phi^{\beta}} .
$$

Здесь $X^{\mu}, \mu=0,1, \ldots, d, d+1,-$ координаты в $(d+1,1)$-мерном объемлюшем ( $d$-бранном) пространстве-времени. Переменные $\phi^{\alpha}, \alpha=0,1, \ldots, d$, описывают протяженный объект; переменные $\phi^{\alpha}$ при $\alpha=1, \ldots, d$ параметризуют $d$-брану, и протяженный объект эволюционирует по $\phi^{0}$.

Действие Намбу-Гото параметризационно инвариантно, и мы покажем, что выборы различных параметризаций ( "параметризации светового конуса" и “декартовой”) приводят к действию для газа Чаплыгина и действию Борна-Инфельда. Эти параметризации выбираются следуюшим образом. В каждом из случаев положим $\left(X^{1}, \ldots, X^{d}\right)$ совпадаюшими с $\left(\phi^{1}, \ldots, \phi^{d}\right)$ и переименуем их в $\mathbf{r}(d$-мерный вектор).

В параметризации светового конуса мы определим $X^{ \pm}$как $\left(X^{0} \pm X^{d+1}\right) / \sqrt{2}$. Переменная $X^{+}$представляет собой переименованную переменную $t$ и отождествляется с $\sqrt{2 \lambda m} \phi^{0}$. Это завершает задание параметризации, а оставшаяся переменная $X^{-}$является функцией $\phi^{0}$ и $\phi$ или после переопределений функцией $t$ и $\mathbf{r}$. Переменная $X^{-}$представляет собой переименованную переменную $\theta(t, \mathbf{r}) / m$, и тогда действие Намбу-Гото (32) в такой параметризации совпадает с действием газа Чаплыгина $\int d t L_{\lambda}$, где $L_{\lambda}$ определяется уравнением (25).

В декартовой параметризации $X^{0}$ представляет собой переименованную переменную $c t$ и отождествляется с $a m c \phi^{0}$. Оставшаяся координата $X^{d+1}$ в объемлющем пространстве, являющаяся функцией $\phi^{0}$ и $\phi$ или, что эквивалентно, функцией $t$ и $\mathbf{r}$, представляет собой переименованную величину $\theta(t, \mathbf{r}) / m c$. Тогда действие Намбу-Гото (32) редуцируется к действию Борна-Инфельда $\int d t L_{a}$, где $L_{a}$ определяется уравнением $(27)$.

Связь с действием Намбу-Гото объясняет происхождение скрытой групшы Пуанкаре в $(d+1,1)$ измерениях в наших двух нелинейных моделях в $(d, 1)$-мерном пространстве-времени: инвариантность Пуанкаре - это то, что остается от репараметризационной инвариантности действия Намбу-Гото после выбора или параметризации светового конуса, или декартовой параметризации. Нелинейная зависимость от поля законов преобразования (12)-(14), (16)-(19), (28)-(31) понятна: она возникает из отождествления некоторых зависимых переменных $\left(X^{\mu}\right)$ с независимыми $\left(\phi^{\alpha}\right)$.

Модель Борна-Инфельда и $(d=1)$-мерный газ Чаплыгина являются полностью интегрируемыми, поскольку оба происходят из струны в двумерном пространстве, а теория Намбу-Гото для такой системы полностью интегрируема [2]. 


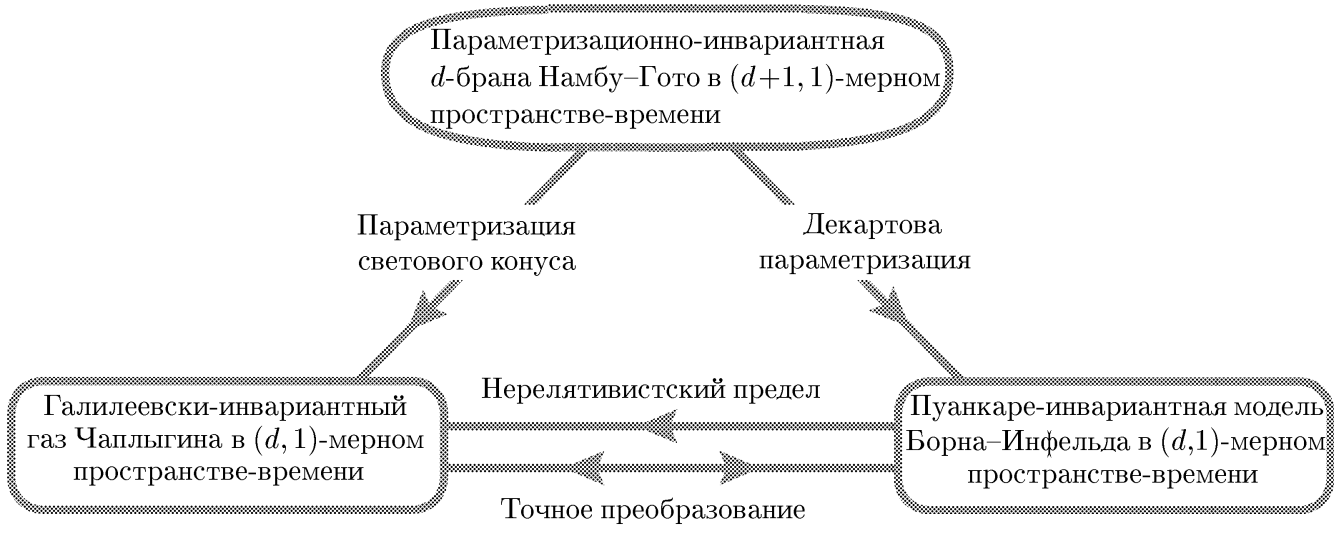

Рис. 3. Дуальности и другие связи между нелинейными уравнениями.

Заметим, что, кроме нерелятивистской наследственности от теории Борна-Инфельда к газу Чапльгина, существует отображение между двумя системами (и между соответствуюшими решениями этих двух систем) вследствие того, что обе системы имеют одного и того же $d$-бранного предка. Отображение достигается переходом от параметризации светового конуса к декартовой параметризации или обратно [3]. А именно это происходит следуюшим образом.

Газ Чаплыгина $\rightarrow$ модель Борна-Инфельда. Задав нерелятивистское решение $\theta_{\mathrm{nr}}(t, \mathbf{r})$, мы определим $T(t, \mathbf{r})$ из уравнения

$$
T+\frac{1}{m c^{2}} \theta_{\mathrm{nr}}(T, \mathbf{r})=\sqrt{2} t .
$$

Тогда релятивистское решение будет иметь вид

$$
\theta_{\mathrm{r}}(t, \mathbf{r})=\frac{1}{\sqrt{2}} m c^{2} T-\frac{1}{\sqrt{2}} \theta_{\mathrm{nr}}(T, \mathbf{r}) .
$$

Модель Борна-Инфельда $\rightarrow$ газ Чаплыгина. Задав релятивистское решение $\theta_{\mathrm{r}}(t, \mathbf{r})$, мы определим $T(t, \mathbf{r})$ из уравнения

$$
T+\frac{1}{m c^{2}} \theta_{r}(T, \mathbf{r})=\sqrt{2} t .
$$

Тогда нерелятивистское решение будет иметь вид

$$
\theta_{\mathrm{nr}}(t, \mathbf{r})=\frac{1}{\sqrt{2}} m c^{2} T-\frac{1}{\sqrt{2}} \theta_{r}(T, \mathbf{r}) .
$$

Все эти связи собраны на рис. 3.

Мы не можем установить связь двух наших нелинейных уравнений с действием Намбу-Гото в отсутствие взаимодействия ни в нерелятивистском $(\lambda=0)$, ни в релятивистском $(a=0)$ случаях: мы не можем исключить $\rho$ в пользу $\theta$, поскольку в отсутствие 
взаимодействия уравнение движения для $\theta$ больше не содержит $\rho$. Эквивалентным образом можно видеть, что $\theta$-лагранжианы $L_{\lambda}(25)$ и $L_{a}(27)$ обрашаются в нуль вместе с $\lambda$ и $а$ соответственно. Действие Намбу-Гото содержит нормировочный множитель, равный натяжению $d$-браны, которое в (32) было положено равным единице. Нерелятивистская и релятивистская свободные модели $(\lambda=0=a)$ в их $\rho$ - $\theta$-формах (5) и (26) соответственно можно поэтому рассматривать как параметризованное описание " $d$-бран без натяжения."

В заключение заметим, что появление групшы Пуанкаре в $(d+1,1)$ измерениях из $(d, 1)$-группы Галилея можно также понять в рамках конструкции типа конструкции Калуцы-Клейна [8].

\section{ЗАКЛЮЧЕНИЕ}

Мы рассмотрели движение нерелятивистской жидкости на примере газа Чапльгина и его релятивистского обобщения, модели Борна-Инфельда, в $(d, 1)$-мерном пространстве-времени. Оба случая обладают скрытой динамической симметрией Пуанкаре. Мы показали, что это результат наследования из действия Намбу-Гото в $(d+1,1)$-мерном пространстве-времени с использованием параметризации светового конуса для газа Чапльпина и декартовой параметризации для модели Борна-Инфельда.

Более того, обшая родословная, связывающая нерелятивистскую и релятивистскую системы, приводит к существованию преобразования между ними, а решение одной системы можно отобразить в решение другой. Полная интегрируемость этих систем является следствием полной интегрируемости теории Намбу-Гото для струн в двумерном пространстве.

Благодарности. Работа выполнена при частичной поддержке Министерства энергетики США (Contract № DE-FC02-94ER40818).

\section{Список литературы}

[1] D. Bazeia, R. Jackiw. Ann. Phys. 1998. V. 270. P. 146.

[2] D. Bazeia. Phys. Rev. D. 1999. V. 59. P. 085007.

[3] R. Jackiw, A. Polychronakos. Faddeev Festschrift. (to appear); Dynamical Poincaré symmetry realized by field-dependent diffeomorphisms. hep-th/9809123; Commun. Math. Phys. (to appear); Fluid dynamical profiles and constants of motion from $d$-branes. hep-th/9902024.

[4] M. Bordemann, J. Hoppe. Phys. Lett. B. 1993. V. 317. P. 315; 1994. V. 325. P. 359; J. Hoppe. Phys. Lett. B. 1994. V. 329. P. 10.

[5] A. Jevicki. Phys. Rev. D. 1998. V. 57. P. 5955.

[6] Y. Nutku. J. Math. Phys. 1987. V. 28. P. 2579; P. Olver, Y. Nutku. J. Math. Phys. 1988. V. 29. P. 1610; M. Arik, F. Neyzi, Y. Nutku, P. Olver, J. Verosky. J. Math. Phys. 1989. V. 30. P. 1338; J. Brunelli, A. Das. Phys. Lett. A. 1997. V. 235. P. 597.

[7] Б. Барбашов, Н. Черников. ЖЭТФ. 1966. Т. 51. С. 658.

[8] M. Hassaine, P. Horvathy. Ann. Phys. (to appear); Field-dependent symmetries of a non-relativistic fluid model. math-ph/9904022. 